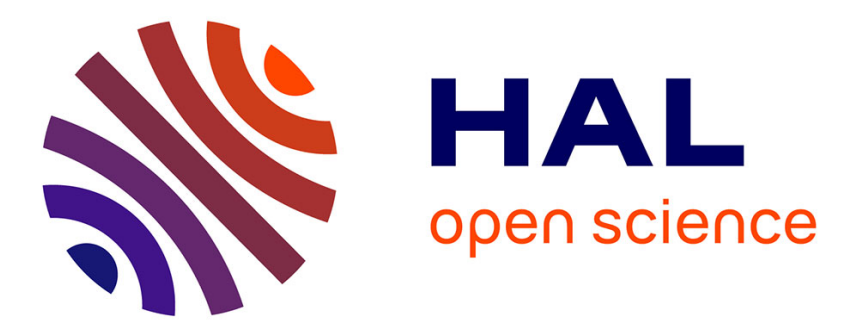

\title{
Dynamic arrest during the spreading of a yield stress fluid drop
}

\author{
Grégoire Martouzet, Loren Jørgensen, Yoann Pelet, Anne-Laure Biance,
} Catherine Barentin

\section{- To cite this version:}

Grégoire Martouzet, Loren Jørgensen, Yoann Pelet, Anne-Laure Biance, Catherine Barentin. Dynamic arrest during the spreading of a yield stress fluid drop. Physical Review Fluids, 2021, 6 (4), pp.044006. 10.1103/PhysRevFluids.6.044006 . hal-03245894

\section{HAL Id: hal-03245894 \\ https://hal.science/hal-03245894}

Submitted on 1 Nov 2021

HAL is a multi-disciplinary open access archive for the deposit and dissemination of scientific research documents, whether they are published or not. The documents may come from teaching and research institutions in France or abroad, or from public or private research centers.
L'archive ouverte pluridisciplinaire HAL, est destinée au dépôt et à la diffusion de documents scientifiques de niveau recherche, publiés ou non, émanant des établissements d'enseignement et de recherche français ou étrangers, des laboratoires publics ou privés. 


\title{
Dynamic arrest during the spreading of a yield stress fluid drop.
}

\author{
Grégoire Martouzet, Loren Jørgensen, Yoann Pelet, Anne-Laure Biance, and Catherine Barentin* \\ ILM, Université de Lyon, Université de Lyon 1 and CNRS, UMR5306, F-69622 Villeurbanne, France
}

(Dated: November 1, 2021)

\begin{abstract}
When a liquid drop is gently deposited on a wetting solid surface, it spreads due to capillary forces until it reaches a thermodynamical equilibrium set by the relative surface energies of the system. We investigate here experimentally the spreading ability of drops made of yield stress fluids, which flow only if the applied stress is above a finite value. We observe that in this case, after a spreading phase, the motion stops and a well-defined contact angle can be measured. This contact angle depends on the rheological properties of the fluid and in particular on its yield stress, on the drop radius and on the hydrodynamic boundary condition at the surface. These results are quantitatively compared to an analysis showing that, due to the yield stress of the fluid, a mechanical equilibrium is indeed reached which does not correspond to the thermodynamical equilibrium.
\end{abstract}

\section{INTRODUCTION}

When a drop of a Newtonian liquid is gently deposited on a smooth substrate, it can spread totally or partially, depending on the nature of the materials (the solid surface, the surrounding gas, the spreading liquid). During the spreading, a so-called contact line is established at the limit of the three phases (here gas, liquid and substrate) and an equilibrium contact angle $\theta_{e}$ is reached. $\theta_{e}$ satisfies both the minimization of the energy of the system and the force balance at the contact line 1 . In the case of a partial spreading on an ideal surface, $\theta_{e}$ is given by Young-Dupré's law: $\cos \theta_{e}=\left(\Gamma_{s g}-\Gamma_{s l}\right) / \Gamma$, where $\Gamma_{s g}, \Gamma_{s l}$ and $\Gamma$ are the interfacial tensions of the solid/gas, solid/liquid and liquid/gas interfaces, respectively. A dynamic regime precedes equilibrium, during which the contact angle $\theta$ decreases and the contact radius $R$ of the drop on the surface increases with time. These dynamics have been extensively studied [2] 6], and the main difficulty has been to model the singularity at the moving contact line, where viscous dissipation diverges. The introduction of a cut-off length and the small-angle and small capillary number approximation lead to the well-established Cox-Voinov law, first derived by Tanner [7, that links the contact line velocity with the dynamic contact angle. However, this commonly accepted description is put into question in the case of complex surfaces [8-10, or complex fluids as investigated here.

Different types of complex fluids can be considered. On the one hand, some non-Newtonian fluids flow like liquids whose viscosity depends on the applied shear rate: a decreasing (resp. increasing) viscosity with the shear rate corresponds to a shear-thinning (resp. shear-thickening) behavior. Spreading dynamics of such shear-thinning systems has been studied theoretically [11] and experimentally [12 and deviates slightly from what is observed for Newtonian liquids. The rheology of the fluid modifies the spreading dynamics, as expected, but not the equilibrium state, which still satisfies surface energy minimization and Young-Dupré's law. On the other hand, fluids with more complex mechanical responses such as foams, concentrated emulsions, polymer microgels, colloidal suspensions and wet granular materials can be encountered. These materials, called yield-stress fluid (YSF), behave like an elastic solid below a critical stress referred to as the yield stress, and above it, they flow like a liquid. This complex behavior that is both liquid and solid is important for many applications in various industries (food, cosmetics, building) [13, 14. More specifically, many processes such as 3D printing, coating or imbibition involve YSFs in contact with surfaces and thus motivate the study of their wetting properties.

In the last decade, studies have examined interfacial properties of YSFs in some situations such as drop formation [15], drop impact [16 18, dip-coating [19]22], capillary adhesion [23, 24] and capillary rise [25]. They highlighted the role of the non-relaxed internal stresses in YSF, leading for example to deviations from the classical Jurin's law in capillary rise [25] or making the measurement of their surface tension difficult [19, 24]. But none have considered the spreading of a YSF drop on a solid surface despite the importance of this case both conceptually and practically. Here we investigate the spreading of a drop made from a YSF on a solid surface and we focus on the influence of the yield stress on the final state, i.e., on the shape of the final drop and on the final contact angle. In particular we address the following question: Does the classical Young-Dupré's law still hold? If not, is it possible to predict it?

First, we describe our experimental procedures for monitoring the spreading of drops made of YSF. Then we present our experimental results on the final contact angle observed as a function of the yield stress, which we 
compare quantitatively to analytical calculations. Finally, a phase diagram showing the different outcomes on the interactions of a YSF drop with a surface is proposed.

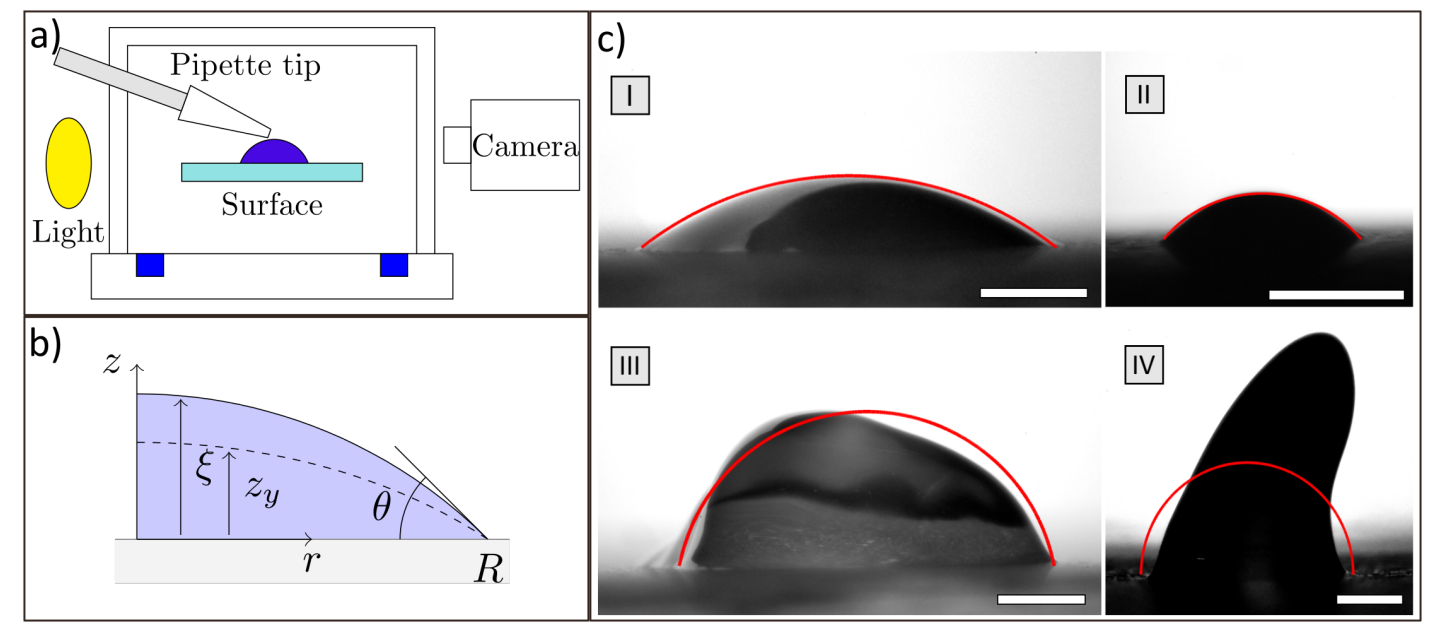

FIG. 1. a) Experimental setup. b) Schematic and notation of a spreading drop: $\theta$ the contact angle, $R$ the radius, $\xi$ the height of the drop and $z_{y}$ the thickness of the sheared zone inside the drop. c) Pictures of drops of various YSFs after spreading on rough surface: (I) Carbopol gel (ETD at $0.3 \%$ ) with a yield stress $\sigma_{y}=6 \mathrm{~Pa}$, (II) and (III) Carbopol gel (ETD at $2 \%$ ) with $\sigma_{y}=35 \mathrm{~Pa}$, (IV) Carbopol gel (U10 at $0.5 \%$ ) with $\sigma_{y}=85 \mathrm{~Pa}$. Fits of the drop shape assuming a spherical cap of a same volume as the drop, are plotted in red. Scale bar corresponds to $1 \mathrm{~mm}$.

\section{EXPERIMENTS AND RESULTS}

Drops made of various fluids are formed at the edge of a pipette tip. Carbopol gels (ETD2050 and U10 from Lubrizol), which are dense suspensions of microgels made of cross-linked polyacrylic acid in water, have been used as model YSF. Different concentrations have been considered to achieve different rheological properties, as reported in Tab. I These rheological properties (yield stress $\sigma_{y}$ and elastic modulus $G^{\prime}$ ) are measured with a rheometer (Anton Paar Physica MCR 301) in a parallel plate geometry (see Supplemental Material at URL for details on rheological measurements). Yield stresses vary between 0 and $75 \mathrm{~Pa}$. The fluid/gas interfacial tension $\Gamma$ is measured using a home-made set-up [24, and is around $60 \mathrm{mN} / \mathrm{m}$, for all the fluids considered. Suspensions of non-crosslinked linear polyacrylic acid (PAA) (from PolySciences), which has no yield stress but similar surfaces properties to Carbopol, have been used for comparison. In the following, Carbopol gels will refer to YSF and PAA suspension will refer to fluid without yield stress.

Drops of different initial radii $\left(R_{0}=0.5-2 \mathrm{~mm}\right)$ are gently deposited, with a velocity below $1 \mathrm{~mm} / \mathrm{s}$, on a hydrophilic microscope glass slide (from $V W R$ ) that is cleaned with an ozone plasma (see Supplemental Material at URL for cleaning procedure). To get smooth substrates, the microscope glass slides are used as received, while for rough ones, the slides are sandblasted. The measured roughness of the substrate is $\sim 20 \mu \mathrm{m}$, always larger than the microstructure of the YSF used, ensuring a no-slip boundary condition at the solid interface [26. As shown in Fig. 1a), the experiments are performed in a sealed box saturated with water vapor to avoid evaporation of the drop. The sealed box is placed in a clean room environment to avoid dust deposits on the microscope glass slides during their transfer from the plasma cleaner to the box.

When the drop touches the substrate, it initially spreads. After a few minutes, the motion stops, which indicates that a final state is reached. A side-view picture is taken with a camera (Nikon d700 with a Navitar Zoom 6000 Lens). As shown in Fig. 1 k), different non-regular drop profiles are observed, depending mainly on the yield-stress value and on the drop radius. From these profiles, we extract the final radius $\left(R_{f}\right)$ and final contact angles at the right and left edges of the drop $\left(\theta_{r}, \theta_{l}\right)$, which are both defined as the greatest slope of the profile $\xi(r)$ on the right (respectively left) side of the drop (see Supplemental Material at URL for the characterisation of contact angles). This extraction is made with a home-made Python code, very similar to ImageJ's Drop Shape Analysis plugin [27. The accuracy on the angle is $5^{\circ}$ and $1 \%$ for the radius. For symmetric shapes as the ones shown in Fig. 1 $\mathrm{k}$ ) (drops I and II), the final contact angle $\left(\theta_{f}\right)$ is defined as the average over $\theta_{l}$ and $\theta_{r}$. When the observed shape is clearly asymmetric, characterized by a difference of left and right angles larger than 10\%, such as the one shown in Fig. 11 ) (drop IV), the final contact angle $\theta_{f}$ is not measured and not compared to the model, which assumes a cylindrical symmetry. 
TABLE I. Properties of the complex fluids: type (two Carbopols and one suspension of PAA), weight concentration $c$, yield stress $\sigma_{y}$, elastic modulus $G^{\prime}$ and surface tension $\Gamma$.

\begin{tabular}{|c|c|c|c|c|}
\hline Type & $c$ (\% wt.) & $\sigma_{y}(\mathrm{~Pa})$ & $G^{\prime}(\mathrm{Pa})$ & $\Gamma(\mathrm{mN} / \mathrm{m})$ \\
\hline Carbopol & 3 & $74 \pm 4$ & $206 \pm 3$ & $55^{\sqrt{a}}$ \\
\hline \multirow[t]{4}{*}{ ETD2050 } & 2 & $35 \pm 2$ & $97 \pm 1$ & $55 \pm 10$ \\
\hline & 1 & $19.0 \pm 1$ & $75 \pm 1$ & $58 \pm 5$ \\
\hline & 0.3 & $6.2 \pm 0.4$ & $34 \pm 0.5$ & $63 \pm 3$ \\
\hline & 0.03 & $\leq 1$ & $6.2 \pm 0.5$ & $63 \pm 2$ \\
\hline Carbopol & 0.25 & $29 \pm 2$ & $170 \pm 1$ & $55 \pm 5$ \\
\hline \multirow[t]{2}{*}{ U10 } & 0.2 & $18 \pm 2$ & $140 \pm 1$ & $55^{*}$ \\
\hline & 0.15 & $4.9 \pm 0.2$ & $53.0 \pm 0.5$ & $55^{*}$ \\
\hline $\mathrm{PAA}$ & 2 & 0 & 0 & $60 \pm 3$ \\
\hline
\end{tabular}

a $*$ corresponds to values of surface tension that have not been measured in 24] (see Supplemental Material at URL for more details).

Variations of the final contact angles $\theta_{f}$ as a function of the final radii $R_{f}$ are reported in Fig. 2 a) for the various complex fluids used, and for smooth and rough substrates. On the contrary to what is observed with Newtonian fluids and to what is expected from the classical thermodynamic description, the final contact angle obtained with the YSFs (Carbopol ETD and U10) depends on the drop size; it increases with $R_{f}$. As a comparison, we also plot $\theta_{f}$ as a function of $R_{f}$ for a PAA suspension, which has no yield stress (see green circles, same figure). As expected for such a system, the final contact angle is almost constant with respect to the drop radius. It is also almost constant with the roughness of the substrate. The second important result is that the final contact angle measured with Carbopol (U10) depends on the substrate roughness (see red squares in Fig. 2a)): rough substrates result in contact angles $\theta_{f}$ larger of at least $20^{\circ}$ for this YSF. Such dependence of the contact angle with the substrate roughness observed with YSF and more importantly the fact that final contact angles are larger on rough substrates than on smooth ones is opposited to the behavior of Newtonian fluids. Indeed for $\theta_{e}<90^{\circ}$, the contact angle measured with Newtonian fluids on a rough substrate is smaller than the one measured on a smooth substrate due to the gain in surface energy, as described by Wenzel's law [28]. Finally, the last important result is the increase of the final contact angle with the yield stress of the fluids, as reported in Fig. $2 \mathrm{~b}$ ), for a given drop size spreading on a rough substrate.

a)

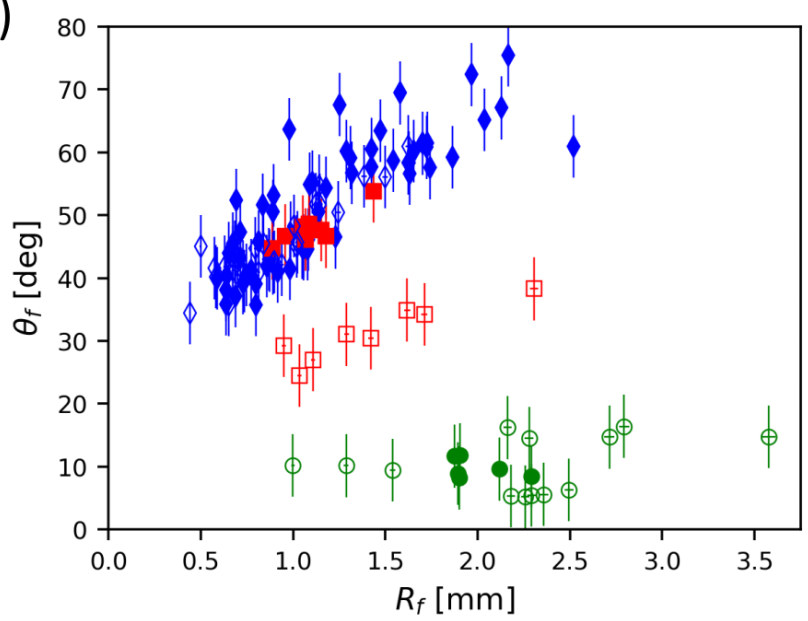

b)

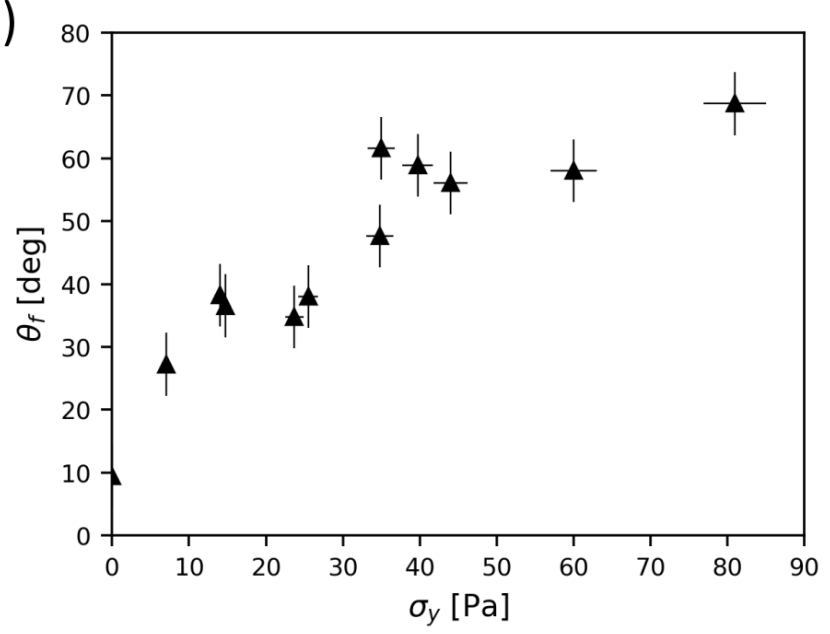

FIG. 2. a) Final contact angle $\theta_{f}$ versus the final radius $R_{f}$ for three fluids defined in Table I Carbopol gel (ETD at $2 \%$ ) with a yield stress $\sigma_{y}=35 \mathrm{~Pa}$ (blue diamonds), Carbopol gel (U10 at $0.25 \%$ ) with a yield stress $\sigma_{y}=29 \mathrm{~Pa}$ (red squares) and PAA suspension (at 2\%) with no yield stress (green circles), on smooth (open symbols) and rough substrates (plain symbols). b) Final contact angle $\theta_{f}$ measured with YSFs on rough substrates as a function of the yield stress $\sigma_{y}$ for a given radius, $R_{f}=1.5 \pm 0.2 \mathrm{~mm}$. 


\section{ANALYSIS AND DISCUSSION}

\section{A. Dynamic of spreading}

Measurements of final contact angle show that the spreading ability of YSFs does not depend only on thermodynamical parameters such as interfacial tensions but also on rheological properties. Therefore dynamical effects have to be considered. We then model the spreading dynamics of a YSF on a solid surface. The flow properties of YSFs are classically described by the empirical Herschel-Bulkley (HB) law: $\dot{\gamma}=0$ if $\sigma \leq \sigma_{y}$, and $\sigma=\sigma_{y}+K \dot{\gamma}^{n}$ if $\sigma>\sigma_{y}$, with $\sigma$ the applied shear stress, $\dot{\gamma}$ the shear rate, $K$ the consistency and $n$ the so-called HB exponent. We then describe the flow profile in the spreading drop, the drop being characterized by its radial height $\xi(r)$. We note $R$ the contact radius of the drop and $V$ the velocity of the contact line, $V=\mathrm{d} R / \mathrm{d} t$. Notations are defined in Fig. $1 \mathrm{~b})$.

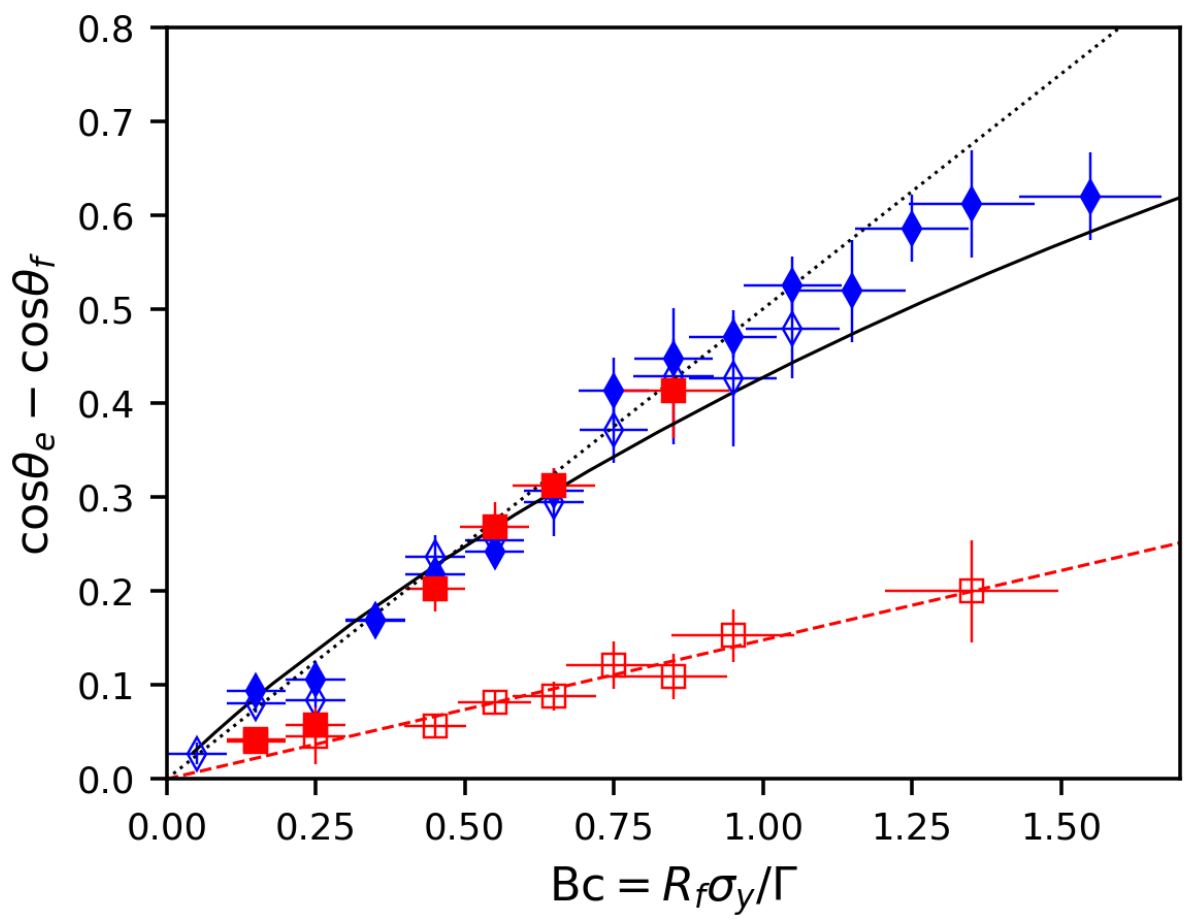

FIG. 3. $\left(\cos \theta_{e}-\cos \theta_{f}\right)$ with $\theta_{e}=10^{\circ}$ as a function of $\mathrm{Bc}=R_{f} \sigma_{y} / \Gamma$ for two YSFs with various concentrations given in Table If Carbopol gel (ETD) (blue diamonds) and Carbopol gel (U10) (red squares), on smooth (open symbols) and rough substrates (plain symbols). Plain line corresponds to the numerical resolution assuming a no-slip boundary condition, black dotted line and red dashed line correspond to the analytical asymptotic limit, assuming a no-slip boundary condition (Eq. 3) and a partial slip boundary condition (Eq. 4 with $V_{s}=0.66 \mathrm{~V}$ ), respectively. A fitting numerical prefactor of $0.50 \pm 0.03$ is obtained in both cases. Each data point corresponds to an average over experimental points in a range of 0.1 for the abscissa.

Following the derivations for Newtonian fluids spreading on an hydrophilic surface [2, we assume that (i) the drops are sufficiently small to neglect gravity, (ii) the dynamical contact angle is small so that the lubrication approximation is satisfied and (iii) inertia is negligible so that the drop has a quasi-static equilibrium shape. The last two assumptions are relevant as soon as the dynamic contact angle is smaller than $30^{\circ}$ [2] and the time after the contact is larger than a few milliseconds [5, 6], respectively.

To determine the spreading dynamics, we first write a momentum balance equation that links the velocity profile within the drop and the pressure gradient. Then, by volume conservation, the pressure gradient is linked to the velocity of the contact line, $V$. Finally, balancing the work done by the capillary force with the viscous dissipation allows us to close the problem.

More precisely, momentum balance links the shear stress $\sigma$ at a position $(r, z)$ within the drop to the radial pressure gradient $A(r)$ as: $\sigma(r, z)=A(r) \cdot(\xi(r)-z)$. Here we assume a free interface. Using the HB law, the relation between the shear rate and the radial velocity $\dot{\gamma}=\partial v_{r} / \partial z$ and assuming a no slip boundary condition, we calculate the velocity 
profile within the drop that reads:

$$
\begin{cases}v_{r}(z)=\frac{n}{n+1}\left(\frac{A}{K}\right)^{\frac{1}{n}} z_{y}^{\frac{1}{n}+1}\left(1-\left(1-\frac{z}{z_{y}}\right)^{\frac{1}{n}+1}\right) & \text { for } 0 \leq z \leq z_{y} \\ v_{r}(z)=\frac{n}{n+1}\left(\frac{A}{K}\right)^{\frac{1}{n}} z_{y}^{\frac{1}{n}+1} & \text { for } z \geq z_{y}\end{cases}
$$

Two distinct zones are identified: a sheared zone near the substrate up to an altitude of $z_{y}(r)=\xi(r)-\sigma_{y} / A(r)$, and a non-sheared zone above that. If $\xi(r) A(r) \leq \sigma_{y}$ then $z_{y}(r)=0$ and the drop stops.

Using Eq. 1, we then apply the flow rate conservation $\int_{0}^{\xi} 2 \pi r v(r, z) d z=Q(r)$ and numerically determine the pressure gradient $A(r)$, assuming a spherical cap for the shape of the drop. Finally, a balance between the capillary and viscous forces allows one to predict the full spreading dynamics. Specifically, the power of the driving capillary force reads $P_{\text {cap }}=2 \pi R \Gamma\left(\cos \theta_{e}-\cos \theta\right) V$ whereas the viscous dissipated power in the drop is:

$$
P_{\text {diss }}=2 \pi \int_{0}^{R} \int_{a}^{\xi} r \sigma \dot{\gamma} \mathrm{d} z \mathrm{~d} r
$$

where $a$ is a cut-off length introduced to regularize the contact line singularity. For Carbopol gels, which are a dense suspension of polymer microgels, we can assume that this cut-off length $a$ is set by the microstructure, i.e. the size of a microgel $(\sim 1 \mu \mathrm{m})$. Note that both powers $\left(P_{\text {diss }}, P_{\text {cap }}\right)$ depend implicitly on time via the geometrical parameters $(R, \theta, \xi)$ which are also time-dependent. Following the methodology of Tanner [7, we assume that the dominant part of the viscous dissipation takes place in the corner near the contact line, in $r=R$, simplifying the problem as a $2 \mathrm{D}$ one on a contour length $2 \pi R$ (see appendix A for details). Taking this assumption, the resulting balance $P_{\text {cap }}(V, t)=P_{\text {diss }}(V, t)$ is solved numerically at each time step that allows to determine $V(t)$. The spreading is then assumed to stop when this balance has no solution any more or when $z_{y}=a$. The first condition corresponds to vanishing spreading velocity and the second corresponds to the situation where the sheared zone has reached the size of a polymer microgel. From this, we can then determine $\theta_{f}$ and $R_{f}$ as a function of the input parameters, such as $\theta_{e}, \Gamma, \sigma_{y}$, and the initial volume of the droplet. Results of $\cos \theta_{e}-\cos \theta_{f}$ as a function of $R_{f} \sigma_{y} / \Gamma$ are reported in Fig. 3

\section{B. End of the spreading}

To get more physical insights, we consider the asymptotic limit near the final state. When the motion is about to stop, the stress contributing to the dissipation, i.e. in the part of the liquid that is flowing, is dominated by the yield stress, so that the stress $\sigma$ in Eq. 2 can be approximated by $\sigma_{y}$. Moreover, in the corner region considered here, we can show that $\int_{a}^{\xi} \dot{\gamma} \mathrm{d} z=V$ (see Appendix A). This implies that the viscous dissipation can be approximated by $P_{\text {diss }} \sim \sigma_{y} V \pi R^{2}$. When balanced with the power done by the capillary forces near the final state, i.e. $\theta=\theta_{f}$, the final contact angle can be derived as:

$$
\cos \theta_{e}-\cos \theta_{f} \sim \frac{\sigma_{y} R_{f}}{\Gamma} .
$$

The characteristic dimensionless number $\mathrm{Bc}=\sigma_{y} R_{f} / \Gamma$ that compares the yield stress and the capillary pressure, known as the Bingham capillary number [29, is then introduced. Eq. 3 predicts that the larger the yield stress and the drop radius, the larger the final contact angle, as observed experimentally in Fig. 2. In the limit of large Bc, the final contact angle $\theta_{f}$ is not defined and the approximations of the model should be revisited, as discussed later.

To test the prediction of the scaling law (Eq. 3), we plot $\left(\cos \theta_{e}-\cos \theta_{f}\right)$ obtained experimentally and numerically as a function of $\mathrm{Bc}=\sigma_{y} R_{f} / \Gamma$, in Fig. 3. The value of the equilibrium contact angle $\theta_{e}$ is here set to $10^{\circ}$ which is the angle measured with the samples exhibiting no yield stress. For small Bingham capillary number $(\mathrm{Bc} \leq 1)$, we find a very good agreement between the scaling law (Eq. 3), the experimental data measured on rough surfaces, and the numerical calculations assuming a no-slip boundary condition. A numerical prefactor of the order of one $(0.50 \pm 0.03)$ is obtained when fitting the data with Eq. 3 (slope of the black dotted line in Fig. 3). We also see that the final contact angles measured with Carbopol gel (ETD) on smooth surfaces (open blue diamonds) are well described by the numerical resolution and the asymptotic limit (Eq. 3) suggesting that a no-slip boundary condition is also satisfied for these experiments. This is not the case for the measurements performed with Carbopol gel (U10); the final contact angles measured on smooth surfaces (open red squares) are much smaller than the ones measured with rough surfaces 


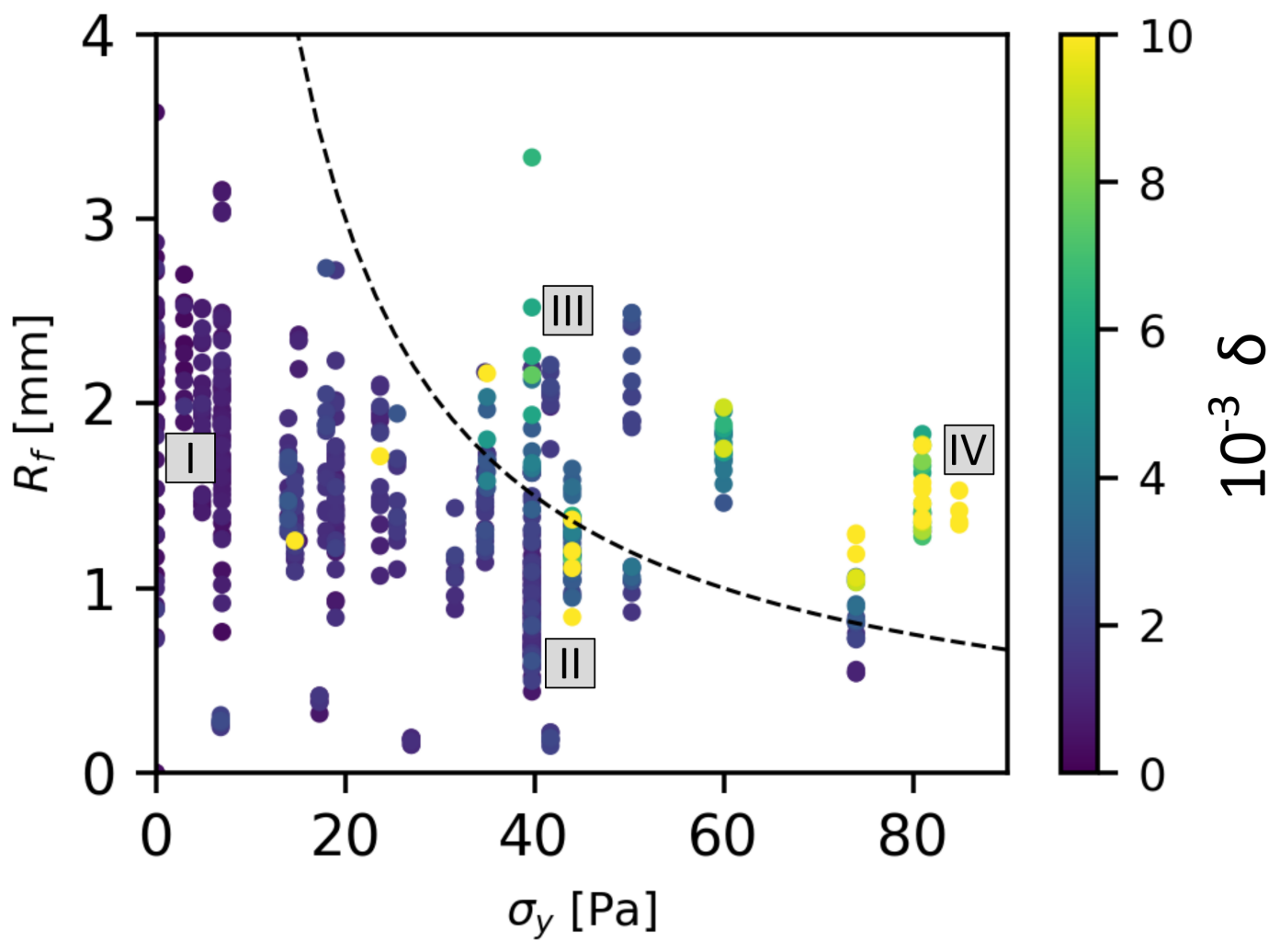

FIG. 4. Schematic of the spreading regimes in the $\left(R_{f}-\sigma_{y}\right)$ phase diagram for all YSFs. The dashed line corresponds to the frontier $R_{f} \sigma_{y}=\Gamma$. The color scale corresponds to the deviation $\delta$ of the final drop shape from a spherical cap as defined in the text (Eq. 5). Roman numbers correspond to the position in this phase diagram of the four pictures from the Fig 1 c).

(plain red squares). We attribute this behavior to the existence of a slip at the wall, observed in different situations but with similar (YSF, smooth substrate) couples [26]. In order to take this new boundary condition into account, we introduce in our model a slip velocity at the solid wall, denoted $V_{s}$. Here, we assume a partial slip boundary condition, i.e., $V_{s}<V$. Friction laws reported with YSFs [14, 26, 30, 31, show that the slip velocity $V_{s}$ essentially depends on the stress at the wall $\sigma_{\text {wall }}=\sigma(r, z=a)$, which a priori depends on the radial position $r$. However, at the end of the spreading, the stress at the wall tends toward the yield stress $\sigma_{y}$ so that, in the corner, $V-V_{s}$ can be considered constant in Eq. 2. The dissipative power then scales as $P_{\text {diss }} \sim \pi R^{2} \sigma_{y}\left(V-V_{s}\right)$ and the balance of powers leads to the following relation:

$$
\cos \theta_{e}-\cos \theta_{f} \sim\left(1-\frac{V_{s}}{V}\right) \frac{\sigma_{y} R_{f}}{\Gamma} .
$$

where $V_{s} / V$ corresponds to the the fraction of slippage in the limit of small velocities. Using this new relation (Eq. (4), a very good agreement is found between the measurements made on smooth surfaces (red open squares in Fig. 3) and the scaling law assuming $V_{s} / V=0.66$ (red dashed line in Fig. 3). Finally the ability of YSFs to slip depends on the microscopic properties of the couple (YSF, substrate), for example on the microgel size and elasticity and on the substrate roughness. In particular, it has been shown [26 that for a given wall stress and fluid yield stress, Carbopol gel (U10) exhibits slip velocities on smooth surfaces much larger than Carbopol gel (ETD). This explains the difference of spreading behavior measured on smooth surfaces.

\section{DISCUSSION}

A crucial outcome from this analysis is that the final contact angle is determined by both the Bingham capillary number Bc and the hydrodynamic boundary conditions at the solid surface. Our analysis is however valid only under three main assumptions we want to discuss here (i) the drop has the shape of a spherical cap (ii) viscous dissipation takes place predominantly near the contact line (iii) gravity is negligible. 
The first main assumption of our analysis is that the shape of the drop is a spherical cap. To test the validity range of this assumption, we fit the drop shapes with a spherical cap of a same volume, as shown in Fig. 1. $)$. To quantify the deviation from the spherical shape, we define the quantity $\delta$ as:

$$
\delta=\frac{1}{R_{f}} \sqrt{\int_{0}^{\pi}\left(\rho_{s c}(\alpha)-\rho(\alpha)\right)^{2} \mathrm{~d} \alpha},
$$

taking polar coordinates in the picture plane (angle $\alpha$, radius $\rho$ and the origin is taken in the center of the drop in the plane of the substrate). $\rho$ and $\rho_{s c}$ correspond to the drop and spherical cap profiles respectively. We then report in Fig. 4 a phase diagram of all experiments performed as a function of the final radius $R_{f}$ and the yield stress $\sigma_{y}$. The color-map corresponds to the value of $\delta$. Pictures of drop profiles (noted I-IV in Fig. 1) are also reported on Fig. 4. One can observe that at large final radius $R_{f}$ or large yield stress $\sigma_{y}, \delta$ is significant so that the deviations from the spherical shape cannot be ignored. A frontier between the spherical and non-spherical regimes can be determined, by comparing the Laplace pressure inside the drop which tends to make it spherical to the yield stress which tends to freeze its shape. This balance reads $\mathrm{Bc}=1$ which is plotted in Fig. 4 (see dashed line). This curve indeed properly delimits the two regions of spherical and non-spherical drops observed experimentally and $\delta$ correctly quantifies the deviation from sphericity. Our full analysis is then valid provided that the Bingham capillary number satisfies Bc $\leq 1$. Outside this limit, the history of drop formation and shape has to be considered. In case of conserved sphericity, note that this limit $\mathrm{Bc} \leq 1$ corresponds also to regimes where the final contact angle is larger than 1 , and then where lubrication approximation fails.

The second main assumption is that viscous dissipation is mainly located near the contact line. Similarly to what is observed for Newtonian fluids, we can indeed estimate that near the contact line, the dissipated power integrated over the thickness of the droplet $\xi$ is diverging. In our complex situation, the liquid is sheared only on a height $z_{y} \ll \xi$, and the dissipated power $p$ per unit length at the contact line then reads, combining Eq. 2 and the complex fluid constitutive law: $p \sim z_{y} K\left(V / z_{y}\right)^{n+1}$. Near the contact line, the height of the droplet tends to zero, so is the sheared zone $z_{y}$, and consequently the dissipation tends to infinity. As classically done for Newtonian fluids, this non-physical divergence is regularized by introducing the microscopic cut-off length $a$ as detailed before. This assumption is then always valid.

The last assumption of our analysis is that gravity is neglected with respect to surface tension. This has been more classically analyzed [1] and is governed by the so-called Bond number which reads Bo $=\rho g R_{f}^{2} / 2 \Gamma$. The criterion Bo $<1$ is always satisfied in our experiments. In a more general prospect, two dimensionless numbers govern the possible motion of a yield-stress fluid drop. The Bingham capillary number Bc that compares yield stress and capillary stress and $\mathrm{Bg}=\sigma_{y} / \rho g h$ that compares yield stress and gravity stress and that we call Bingham gravity number in the following. Here $h$ stands for the height of the drop which depends both on the drop size $R$ and on the contact angle $\theta$. To simplify its expression, we can identify $h$ to the initial radius of the drop $R_{0}$ and compare both numbers $\mathrm{Bc}$ and $\mathrm{Bg}$ at the beginning of the spreading. In the limit of small (respectively large) drop size, the Bingham capillary number Bc (respectively Bingham gravity number $\mathrm{Bg}$ ) is smaller than 1 and the motion is induced by capillary stresses (respectively gravity stresses). Moreover, for sufficiently large yield stresses $\left(\sigma_{y}>\sqrt{\Gamma \rho g} \sim 25 \mathrm{~Pa}\right.$ ), there is a range of drop sizes, $\Gamma / \sigma_{y}<R_{0}<\sigma_{y} / \rho g$, for which motion is impossible. In our experiments, the range of drop radii and heights explored is such as the Bond number is smaller than 1 and the Bingham gravity number is larger than 1 . This implies that the spreading of the drop, if it exists, is solely due to capillary forces.

Finally a last point interesting to discuss is the possible role of the elasticity on the spreading of YSF drop. Depending on the solicitation, a YSF can indeed behave as a visco-plastic fluid or an elastic solid. At the beginning of the spreading, the capillary stress is so high compared to the yield stress that the YSF flows like a liquid [14. But as the spreading goes on, the capillary stress decreases until reaching the yield stress that leads to the drop arrest. At the end of the spreading, most of the drop behaves like a solid under stress. It is then possible to estimate the stored elastic energy in the solid part of the drop and to compare it to the capillary energy (see Appendix B for more details). Quantitative comparison shows that for Bingham-capillary number $B_{\mathrm{c}}<1$, and for YSFs with $\sigma_{y} / G^{\prime}<1$, elasticity plays a negligible role. The spreading of a YSF drop allows then to explore mainly its visco-plastic behavior.

\section{CONCLUSION}

We measure and model the spreading of drops made of YSFs deposited on a wetting solid surface, induced by capillary forces. We observe that after a few minutes, the motion stops and the shape of the drop is frozen. If the Bingham capillary number of the experiment is smaller than 1, the drop takes the shape of a spherical cap. In this limit, the spreading motion stops at a well defined radius and contact angle. We show that the final contact angle does not depend only on the thermodynamical parameters such as interfacial energies but also on the dynamic parameters 
such as the drop radius, the YSF flow properties and the hydrodynamic boundary condition. With a full analysis of the flow profile in the drop, we predict the contact angle of the arrested state, which corresponds very well to experimental observations. Using a quantitative asymptotic analysis, we show that the final contact angle is directly set by the Bingham capillary number and the hydrodynamic boundary conditions. This macroscopic dynamic arrest then depends on the history of the drop spreading. Here several questions arise concerning the appearance of contact angle hysteresis [32, the effect of the impact velocity on the spreading [16, or the existence of a precursor film at the molecular level [33].

G. Martouzet acknowledges ENS Paris-Saclay for funding. We thank Agnès Piednoir for the measurements of surface roughness by AFM and optical profilometer, Gilles Simon for the conception of the experimental set-up and Yedhir Mezache for interesting discussions.

\section{ACKNOWLEDGMENTS} and we detail the two asymptotic limits: the beginning and the end of the spreading.

\section{Appendix A: Modelisation}

In this section, we detail the calculations of the spreading dynamics of the drop until the dynamical arrest is reached

\section{General approach and main assumptions}

As mentioned previously, the fluid rheology is assumed to be described by the HB law:

$$
\begin{cases}\dot{\gamma}=0 & \text { if } \quad \sigma \leq \sigma_{y} \\ \sigma=\sigma_{y}+K \dot{\gamma}^{n} & \text { if } \quad \sigma>\sigma_{y}\end{cases}
$$

where $\sigma_{y}$ is the yield stress, $K$ the consistency and $n$ the HB exponent. The geometry of the spreading drop is assumed to be a spherical cap so it is axisymmetric as drawn in Fig. 1p). We also assume that the spreading dynamics and the dynamical arrest are set by a balance between the capillary force and the viscous dissipation. The power injected by the driving capillary force writes $P_{\text {cap }}=2 \pi R \Gamma\left(\cos \theta_{e}-\cos \theta\right) V$, and the power dissipated by viscosity is given by $P_{\text {diss }}=2 \pi \int_{0}^{R} \int_{a}^{\xi} r \sigma \dot{\gamma} \mathrm{d} z \mathrm{~d} r$ where $\xi(r)$ is the height of the drop and $a$ is a cut-off length that we introduce in order to regularize the divergence of the viscous dissipation at the contact line. In the case of YSFs, the cut-off length is set by the microstructure composing the complex fluids. Here, for Carbopol gels, we assume that the cut-off length is given by the size of a polymer microgel, typically of the order of $1 \mu \mathrm{m}$. Balancing the two powers results in:

$$
\left(\cos \theta_{e}-\cos \theta\right) V \Gamma R=\int_{0}^{R} \int_{a}^{\xi} r \sigma \dot{\gamma} \mathrm{d} r \mathrm{~d} z
$$

To calculate the viscous power, it is necessary to determine the shear stress and the velocity everywhere inside the drop during its spreading. The stress results from the momentum balance, and in the lubrication approximation, it reads:

$$
\sigma(r, z)=A(r)(\xi(r)-z)
$$

where $A$ is the pressure gradient created by the capillary forces, and $\xi$ is the liquid-air interface position as shown in Fig. 1 b). The stress is maximum at the wall and decreases down to zero at the air-liquid interface. From the knowledge of the stress (Eq. A3) and using the HB law (Eq. A1), we can obtain the shear rate everywhere inside the drop:

$$
\begin{cases}\dot{\gamma}(z)=\left(\frac{\sigma(z)-\sigma_{y}}{K}\right)^{1 / n}=\left(\frac{A}{K}\right)^{1 / n}\left(z_{y}-z\right)^{1 / n} & \text { for } 0 \leq z \leq z_{y} \\ \sigma(z) \leq \sigma_{y} \text { and } \dot{\gamma}=0 & \text { for } z \geq z_{y}\end{cases}
$$

where $z_{y}$ is the vertical position inside the drop where the shear stress is equal to the yield stress. By definition, $\sigma\left(z_{y}\right)=\sigma_{y}$ and $z_{y}$ verifies:

$$
z_{y}(r)=\xi(r)-\frac{\sigma_{y}}{A(r)}
$$


Note that the height of the drop $\xi$ and the pressure gradient $A$ depend both on the radial position $r$ so that $z_{y}$ is also a function of $r$, making the calculation of the dissipative power more difficult than for a Newtonian fluid. Finally, $z_{y}$ delineates two regions inside the drop: a liquid-like region near the wall and a solid-like region above it. Indeed, for $z<z_{y}, \dot{\gamma}>0$ and the YSF is sheared and for $z>z_{y}, \dot{\gamma}=0$, the YSF is not sheared and it flows like a plug, as expected for YSFs [14.

We can now integrate the shear rate (Eq. A4) with respect to $z$ and obtain the velocity profile inside the drop:

$$
\begin{cases}v_{r}(z)=\frac{n}{n+1}\left(\frac{A}{K}\right)^{\frac{1}{n}} z_{y}^{\frac{1}{n}+1}\left(1-\left(1-\frac{z}{z_{y}}\right)^{\frac{1}{n}+1}\right) & \text { for } 0 \leq z \leq z_{y} \\ v_{r}(z)=\frac{n}{n+1}\left(\frac{A}{K}\right)^{\frac{1}{n}} z_{y}^{\frac{1}{n}+1} & \text { for } z \geq z_{y}\end{cases}
$$

Here we assume a no-slip boundary condition at the solid surface. Such boundary condition is indeed valid for YSF flowing on rough surfaces [14. From the knowledge of the velocity profile, we can also calculate the mean velocity which depends on the radial position $r$ such as:

$$
V_{m}(r)=\frac{1}{\xi(r)} \int_{0}^{\xi(r)} v_{r}(z) \mathrm{d} z
$$

In particular, near the contact line, we have $\lim _{r \rightarrow R} V_{m}(r)=V$.

To solve the spreading dynamics and find $R(t)$, it is necessary first to determine the pressure gradient $A(r)$ by using the mass conservation and then to integrate it in the balance of the powers. This has to be done numerically (see Supplemental Material at URL for details on the numerical resolution). Finally, the link between the angle $\theta(t)$ and $R(t)$ is given by the conservation of the drop volume, assuming a spherical-cap shape.

\section{Asymptotic limits}

Two asymptotic limits can be solved analytically: 1- the beginning of the spreading characterised by high shear rates and dominated by the viscous properties of the YSF and 2- the end of the spreading characterised by small shear rates and dominated by the plasticity of the YSF. High or low shear rates are defined with respect to $\dot{\gamma}^{*}=\left(\sigma_{y} / K\right)^{1 / n}$ for which the two terms of the HB law are equal: $\sigma_{y}=K \dot{\gamma}^{n}$.

At the beginning of the spreading, the shear rate is large compared to $\dot{\gamma}^{*}$ such as the yield stress $\sigma_{y}$ can be neglected with respect to the viscous stress $K \dot{\gamma}^{n}$ in Eq. A1. So the YSF can be considered as a shear-thinning system without yield stress. The entire problem can be solved analytically and the spreading dynamics follows: $R(t) \propto t^{p}$ with $p=n /(3 n+7)$, known as the Starov's law [11.

At the end of the spreading, the shear rate is small compared to $\dot{\gamma}^{*}$ such as the viscous stress $K \dot{\gamma}^{n}$ can be neglected with respect to the yield stress $\sigma_{y}$. The stress inside the drop is then dominated by the yield stress. Consequently, the power associated to the viscous dissipation can be approximated to: $P_{\mathrm{diss}}=2 \pi \int_{0}^{R} \int_{a}^{\xi} r \sigma \dot{\gamma} \mathrm{d} z \mathrm{~d} r \simeq 2 \pi \sigma_{y} \int_{0}^{R} r \int_{a}^{\xi} \dot{\gamma} \mathrm{d} z \mathrm{~d} r$, where $\int_{a}^{\xi} \dot{\gamma} \mathrm{d} z=v(\xi)-v(a)$ corresponds to the difference of velocities between the top and the bottom of the drop. Note that $v(\xi)=v\left(z_{y}\right)$ due the existence of the unsheared region above $z_{y}$, so that $\int_{a}^{\xi} \dot{\gamma} \mathrm{d} z=v\left(z_{y}\right)-v(a)$.

For YSFs, two boundary conditions (BC) can be encountered: no-slip or partial-slip BCs [14, 30]. In the case of a no-slip BC, $v(a)=0$ and the dissipative power writes $P_{\text {diss }} \simeq 2 \pi \sigma_{y} \int_{0}^{R} r v\left(z_{y}(r)\right) \mathrm{d} r$.

To go one step further, we assume, as it is done for Newtonian liquids when deriving the Tanner's law [7, that the viscous dissipation dominates in the corner of the drop near the contact line, where regularization is required to avoid divergence of the viscous stress. Using Eq. A7 near the contact line, we can show that the velocity inside the drop and the spreading velocity $V$ are linked by:

$$
\lim _{r \rightarrow R} \frac{1}{\xi(r)} \int_{0}^{\xi(r)} v_{r}(z) \mathrm{d} z=V
$$

The integral over the velocity on the left hand side of Eq. A8 can be calculated knowing the velocity profile inside the drop (Eq. A6): $\int_{0}^{\xi(r)} v_{r}(z) \mathrm{d} z=\left(\xi-z_{y}\right) v\left(z_{y}\right)+\int_{0}^{z_{y}} v(z) \mathrm{d} z$. In the asymptotic limit, the sheared region vanishes and becomes negligible compared to the height of the drop, i.e., $z_{y}<<\xi$. This implies that $\lim _{\sigma \rightarrow \sigma_{y}} v\left(z_{y}\right)=V$ near the contact line. The integral over the radial position in the dissipative power, $\int_{0}^{R} r v\left(z_{y}(r)\right) \mathrm{d} r$ can be then simplified 
and approximated to a term proportional to $V R^{2}$. The dissipative power then reads:

$$
P_{\text {diss }} \simeq b \sigma_{y} R^{2} V
$$

where $b$ is a dimensionless constant that takes into account the complexity of the calculation of the integral over the geometry of a spherical cap. The balance of the capillary power and the dissipative power at the end of the spreading leads then to:

$$
\cos \theta_{e}-\cos \theta_{f} \simeq c \frac{\sigma_{y} R_{f}}{\Gamma}
$$

where $c=b /(2 \pi)$ is also a dimensionless constant. This asymptotic limit predicts that the distance to the equilibrium $\left(\cos \theta_{e}-\cos \theta_{f}\right)$ increases linearly with the Bingham capillary number $\mathrm{Bc}=\left(\sigma_{y} R_{f}\right) / \Gamma$. This prediction is in very good agreement with what is found experimentally (see Fig. 3). The fit with Eq. A10 of the data, obtained with YSFs spreading on rough surfaces for which no-slip BC is expected leads to $c=0.5$ (black dotted line in Fig. 3).

In the case of a partial-slip BC, the velocity $v(a)$ is non zero. By definition of the cut-off length $a$, it corresponds to the velocity of the first layer of polymer microgel and corresponds to the slip velocity $V_{s}$ which is measured experimentally [26, 30. Measurements of slip velocity $V_{s}$ showed that it depends essentially on the stress at the wall $\sigma_{\text {wall }}, V_{s}\left(\sigma_{\text {wall }}\right)$ [14]. The dissipative power is then:

$$
P_{\text {diss }} \simeq 2 \pi \sigma_{y} \int_{0}^{R} r\left(v\left(z_{y}\right)-V_{s}\left(\sigma_{\text {wall }}\right)\right) \mathrm{d} r
$$

According to Eq. A3, stress at the wall has the following expression $\sigma_{\text {wall }}=A(r) \zeta(r)$ and $a$ priori depends on the radial position $r$. However, at the end of the spreading, the wall stress tends to the yield stress value and becomes almost independent of $r$, as shown in Fig. 5. In the asymptotic limit, we can then consider that: $\sigma_{\text {wall }} \simeq \sigma_{y}$ simplifying the calculation of the dissipative power (Eq. A11).

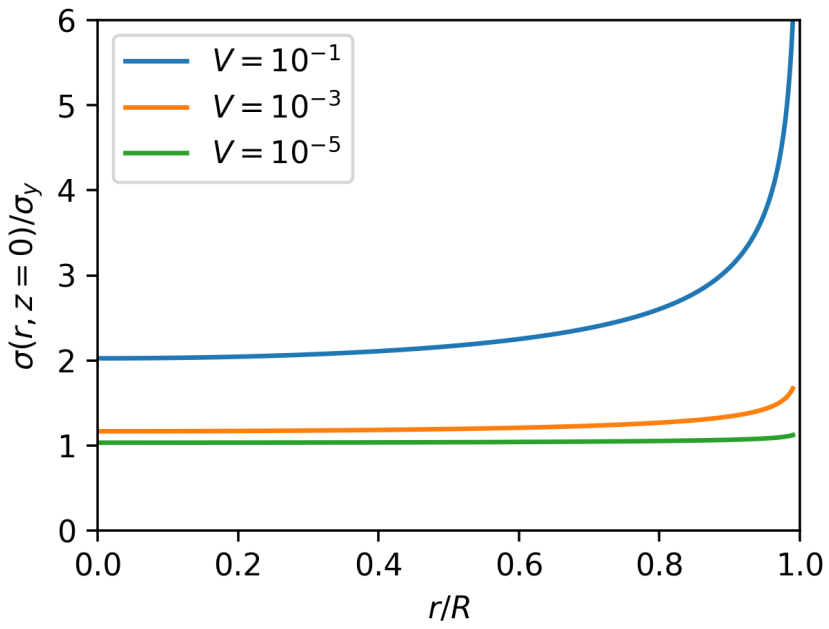

FIG. 5. Stress at the wall $\sigma(z=0) / \sigma_{y}$ as a function of the radial position for three velocities: $10^{-1}$ (blue), $10^{-3}$ (orange) and $10^{-5}$ (green). The velocity here is dimensionless, divided by $R_{0}\left(K / \sigma_{y}\right)^{1 / n}$. As the velocity decreases, the wall stress tends to the yield stress. The data are calculated numerically (see Supplemental Material at URL for more details on the numerical resolution).

Finally, as previously done, we assume that the whole dissipation inside the moving drop is dominated by the dissipation inside the corner near the contact line. Using this assumption, we can show that $\lim _{\sigma \rightarrow \sigma_{y}}\left(v\left(z_{y}\right)-V_{s}\left(\sigma_{\text {wall }}\right)\right)=$ $V-V_{s}$. By definition of the dissipative power, $V_{s}<V$ which corresponds to a partial slip boundary condition. Following the same derivation as in the case of a no-slip BC, we find that:

$$
P_{\text {diss }} \simeq b \sigma_{y} R_{f}^{2}\left(V-V_{s}\right)
$$

The balance of powers then leads to:

$$
\cos \theta_{e}-\cos \theta_{f}=c\left(1-\frac{V_{s}}{V}\right) \frac{\sigma_{y} R_{f}}{\Gamma}
$$


where $c$ is the dimensionless constant defined in Eq. A10 and $V_{s} / V$ is the fraction of slip at the end of the spreading. This asymptotic limit is plotted in Fig. 3 (see red dotted line with $c=0.5$ ). By imposing $V_{s} / V=0.66$, this asymptotic limit describes well the experimental data obtained with Carbopol gels (U10) flowing over smooth surfaces (red open squares) for which a partial slip BC is expected. Concerning the slip velocity, note that we consider a partial slip boundary condition, $V_{s} / V<1$, which implies that the slip velocity vanishes when $V$ tends to 0 , i.e., when the spreading stops.

\section{Appendix B: Role of the elasticity?}

We discuss here the possible role of the elasticity $\left(G^{\prime}\right)$ on the spreading of YSF drops. Therefore, we first estimate the elastic energy stored in the arrested drop and compare it to the capillary energy. Second, we show measurements of final contact angles performed with Carbopol gels of same yield stress $\sigma_{y}$ and different elastic modulus $G^{\prime}$.

\section{Stored elastic energy}

Following the derivation of the velocity profile inside the drop, we show that the drop is composed by a sheared region close to the wall and a non-sheared region for $z>z_{y}$ (see Eq. A6). This non-sheared region behaves like a solid under stress $\left(\sigma\left(z_{y}\right)=\sigma_{y}\right)$, which stores elastic energy. At the end of the spreading, the non-sheared region invades the drop and the stored elastic energy is maximum. Assuming a linear elastic response, if we note $\epsilon$ the local deformation, the elastic energy density is $\sigma=G^{\prime} \epsilon$. Balancing it with the local stress (to replace the value of $\epsilon=\sigma / G^{\prime}$ ) and after integration, we can estimate the maximum stored elastic energy:

$$
E_{\text {elastic }}=\frac{1}{6} \frac{\sigma_{y}^{2}}{G^{\prime}} V
$$

where $V$ is the volume of the drop. This energy has to be compared to the capillary energy that is bounded by:

$$
E_{\text {capillary }}=\pi \Gamma R_{f}^{2}
$$

Assuming a spherical cap shape, we can show that:

$$
\frac{E_{\text {elastic }}}{E_{\text {capillary }}}=\frac{1}{18} B_{c} \frac{\sigma_{y}}{G^{\prime}} f\left(\theta_{f}\right)
$$

where $f$ depends on the contact angle:

$$
f(\theta)=(1-\cos \theta)^{2}(2+\cos \theta) / \sin ^{3} \theta
$$

and ranges from 0.2 to 1.2 for $10^{\circ}<\theta<70^{\circ}$.

This estimation shows that elasticity plays a even smaller role as $B_{c}$ and $\sigma_{y} / G^{\prime}$ are small compared to one.

For typical data as the one shown in Fig. 1 1 ) (picture II) with Carbopol EDT at $2 \%\left(\sigma_{y}=35 \mathrm{~Pa}, G^{\prime}=97 \mathrm{~Pa}\right)$, a final radius of $R_{f}=1 \mathrm{~mm}$, a final contact angle $\theta_{f}=45^{\circ}$, we find that $E_{\text {elastic }} / E_{\text {capillary }} \sim 1 \%$ which suggests that the stored elastic energy is negligible in the spreading.

\section{Influence of the shear elastic modulus ?}

In order to investigate the role of the elastic modulus on the spreading of YSF, we also performed experiments with YSF of similar yield stress values and different elastic modulii. More precisely, we used two carbopol gels (ETD 2050 at $1 \%$ and $\mathrm{U} 10$ at $0.2 \%$ ) whose yield stresses are $\sigma_{y}=18$ and $19 \mathrm{~Pa}$ respectively and whose elastic moduli are $G^{\prime}=75$ and $140 \mathrm{~Pa}$. Although the elastic moduli differ by a factor 2, the final contact angles are identical as shown on Fig 6 (see blue and red data). These results confirm that the elasticity $\left(G^{\prime}\right)$ has a negligible influence compared to the plasticity $\left(\sigma_{y}\right)$ on the spreading of YSF drop.

[1] P.-G. de Gennes, F. Brochard-Wyart, and D. Quéré, Capillarity and Wetting Phenomena: Drops, Bubbles, Pearls, Waves (Springer, 2004). 


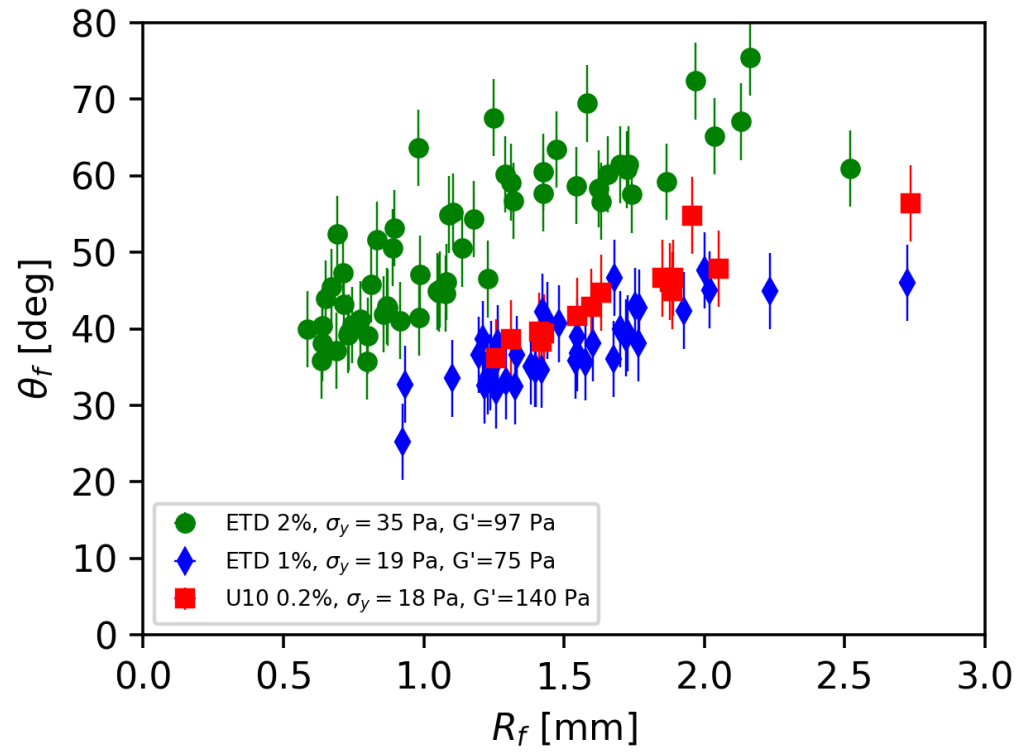

FIG. 6. Final contact angles measured with three different Carbopol gels as a a function of the final radius. Blue and red data have been obtained with YSF of similar $\sigma_{y}$ but different $G^{\prime}$. Blue and green data have been obtained with YSF of similar $G^{\prime}$ but different $\sigma_{y}$.

[2] R. I. Tanner, A theory of die-swell, J. Polym. Sci. A-2 8, 2067 (1970).

[3] O. Voinov, Hydrodynamics of wetting, Fluid dynamics 11, 714 (1976).

[4] P.-G. De Gennes, Wetting: statics and dynamics, Reviews of modern physics 57, 827 (1985).

[5] A.-L. Biance, C. Clanet, and D. Quéré, First steps in the spreading of a liquid droplet, Phys. Rev. E 69, 016301 (2004).

[6] A. Eddi, K. G. Winkels, and J. H. Snoeijer, Short time dynamics of viscous drop spreading, Physics of fluids 25, 013102 (2013).

[7] L. Tanner, The spreading of silicone oil drops on horizontal surfaces, Journal of Physics D: Applied Physics 12, 1473 (1979).

[8] A. Lafuma and D. Quéré, Superhydrophobic states, Nature materials 2, 457 (2003).

[9] H. Perrin, R. Lhermerout, K. Davitt, E. Rolley, and B. Andreotti, Defects at the nanoscale impact contact line motion at all scales, Physical review letters 116, 184502 (2016).

[10] B. Andreotti and J. H. Snoeijer, Statics and dynamics of soft wetting, Annual Review of Fluid Mechanics 52 (2020).

[11] V. Starov, A. Tyatyushkin, M. Velarde, and S. Zhdanov, Spreading of non-newtonian liquids over solid substrates, Journal of Colloid and Interface Science 257, 284 (2003).

[12] S. Rafaï and D. Bonn, Spreading of non-newtonian fluids and surfactant solutions on solid surfaces, Physica A: Statistical Mechanics and its Applications 358, 58 (2005).

[13] R. Mezzenga, P. Schurtenberger, A. Burbidge, and M. Michel, Understanding foods as soft materials, Nature Mater. 4, $729(2005)$.

[14] D. Bonn, M. M. Denn, L. Berthier, T. Divoux, and S. Manneville, Yield stress materials in soft condensed matter, Reviews of Modern Physics 89, 035005 (2017).

[15] G. German and V. Bertola, Formation of viscoplastic drops by capillary breakup, Physics of fluids 22, 033101 (2010).

[16] L.-H. Luu and Y. Forterre, Drop impact of yield-stress fluids, Journal of Fluid Mechanics 632, 301 (2009).

[17] G. German and V. Bertola, Impact of shear-thinning and yield-stress drops on solid substrates, Journal of Physics: Condensed Matter 21, 375111 (2009).

[18] G. German and V. Bertola, The spreading behaviour of capillary driven yield-stress drops, Colloids and Surfaces A: Physicochemical and Engineering Aspects 366, 18 (2010).

[19] J. Boujlel and P. Coussot, Measuring the surface tension of yield stress fluids, Soft Matter 9, 5898 (2013).

[20] M. Maillard, J. Bleyer, A. Andrieux, J. Boujlel, and P. Coussot, Dip-coating of yield stress fluids, Physics of Fluids 28, $053102(2016)$.

[21] A. Gans, E. Dressaire, B. Colnet, G. Saingier, M. Z. Bazant, and A. Sauret, Dip-coating of suspensions, Soft matter 15, $252(2019)$.

[22] W. J. Smit, C. Kusina, J.-F. Joanny, and A. Colin, Stress field inside the bath determines dip coating with yield-stress fluids in cylindrical geometry, Physical review letters 123, 148002 (2019).

[23] Q. Barral, G. Ovarlez, X. Chateau, J. Boujlel, B. Rabideau, and P. Coussot, Adhesion of yield stress fluids, Soft Matter 6, $1343(2010)$. 
[24] L. Jørgensen, M. Le Merrer, H. Delanoë-Ayari, and C. Barentin, Yield stress and elasticity influence on surface tension measurements, Soft Matter 11, 5111 (2015).

[25] B. Géraud, L. Jørgensen, L. Petit, H. Delanoë-Ayari, P. Jop, and C. Barentin, Capillary rise of yield-stress fluids, Europhys. Lett. 107, 58002 (2014).

[26] J. Péméja, B. Géraud, C. Barentin, and M. Le Merrer, Wall slip regimes in jammed suspensions of soft microgels, Phys. Rev. Fluids 4, 033301 (2019)

[27] A. Stalder, G. Kulik, D. Sage, L. Barbieri, and P. Hoffmann, A snake-based approach to accurate determination of both contact points and contact angles, Colloids And Surfaces A: Physicochemical And Engineering Aspects 286, 92 (2006).

[28] R. N. Wenzel, Resistance of solid surfaces to wetting by water, Industrial \& Engineering Chemistry 28, 988 (1936).

[29] V. Bertola, Wicking with a yield stress fluid, J. Phys. Condens. Matter 21, 035107 (2009).

[30] S. P. Meeker, R. T. Bonnecaze, and M. Cloitre, Slip and flow in soft particle pastes, Phys. Rev. Lett. 92, 198302 (2004).

[31] X. Zhang, E. Lorenceau, P. Basset, T. Bourouina, F. Rouyer, J. Goyon, and P. Coussot, Wall slip of soft-jammed systems: A generic simple shear process, Phys. Rev. Lett. 119, 208004 (2017).

[32] H. Eral, D. 't Mannetje, and J. Oh, Contact angle hysteresis: a review of fundamentals and applications., Colloid and Polymer Science 291, 247-260 (2013).

[33] M. N. Popescu, G. Oshanin, S. Dietrich, and A. Cazabat, Precursor films in wetting phenomena, Journal of Physics: Condensed Matter 24, 243102 (2012). 